\title{
Detection of localized overload during the bedding of patient as an alarm system for decubitus prophylaxes
}

Böhringer S., University of Applied Sciences and Arts Northwestern Switzerland, Muttenz, Switzerland Suter, Ch., University of Applied Sciences and Arts Northwestern Switzerland, Muttenz, Switzerland Jeker, M., University of Applied Sciences and Arts Northwestern Switzerland, Muttenz, Switzerland Hradetzky, D., University of Applied Sciences and Arts Northwestern Switzerland, Muttenz, Switzerland (david.hradetzky@fhnw.ch, corresponding author)

\section{Introduction}

During time-consuming surgical procedures the load applied on patient's skin-tissue is an important factor for developing decubitus ulcer. This may due to a forgotten wire or wrinkled bed sheet beneath the patient, leading to localized high pressure, and, depending on the duration, enhancing the risk of suffering from decubitus [1]. For being able to detect the localized load, usually a high resolving pressure sensor array is necessary.

Goal of this project is the proof of simple low resolution alarm sensor concept, capable to detect a localized overload affecting only small parts or the sensor surface, and working independently from the size of the affected region.

\section{Device}

The sensor consists of two electrically conductive layers, and an isolating layer in-between. The isolating layer is formed in a structured shape, including wholes. Applying pressure on the top, the layers will deform, causing an electrical connection between the both conductive layers.

\section{Results}

We elaborated, realized and proofed the concept for two different sensor configurations.

In first configuration the isolating layer is formed by a structure of intertwines columns, exhibiting a shift in the threshold pressure value by increasing the affected region. Second configuration, based on a honeycombed isolating layer, provides a sensor response independent from the affected region.

For detailed insight to sensor response and to optimize the isolating structures, the devices were simulated using FEM (COMSOL $\AA)$. Adjustment of threshold value can be realized either by modifying the isolating structure or material.

\section{Conclusion}

A proof of concept for a pressure sensitive, area-independent threshold switch was realized in two different sensor designs. The simple set up of the device makes it feasible to be integrated within large area single use devices or textiles, and therefore we expect this concept to be suitable to be used on OR-tables or hospital beds as an alarm system for decubitus prophylaxes.

[1] J. B. Reswick and J. E. Rogers, "Experience at Rancho Los Amigos Hospital with devices and techniques to prevent pressure sores," in Bed Sores Biomechanics, R. M. Kenedi, J. M. Cowden, and J. T. Scales, Eds. London: University Park Press, 1976, pp. 301-310. 